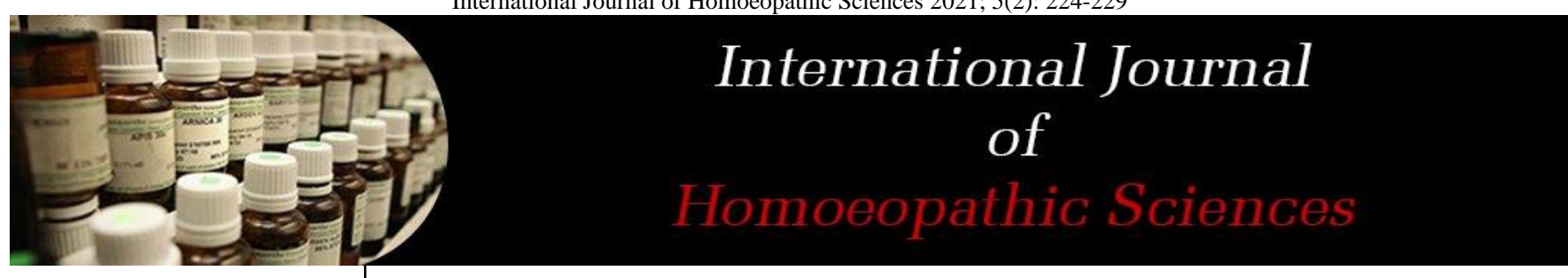

E-ISSN: $2616-4493$

P-ISSN: 2616-4485

www.homoeopathicjournal.com

IJHS 2021; 5(2): 224-229

Received: xx-02-2021

Accepted: xx-03-2021

Dr. Siddabathina Uma Devi BHMS, (MD), PG Part-2, Department of Homoeopathic Materia Medica, Guru Mishri Homoeopathic Medical

College, Maharashtra, India

\section{Dr. Hulekar}

HOD, Department of

Homoeopathic Materia

Medica, Guru Mishri

Homoeopathic Medical

College, Maharashtra, India

Dr. Sohera Talat

PG Guide, Guru Mishri

Homoeopathic Medical

College, Maharashtra, India
Corresponding Author: Dr. Siddabathina Uma Devi BHMS, (MD), PG Part-2, Department of Homoeopathic Materia Medica, Guru Mishri Homoeopathic Medical College, Maharashtra, India

\title{
An overview of epilepsy with a note on homoeopathic approach
}

Dr. Siddabathina Uma Devi, Dr. Hulekar and Dr. Sohera Talat

DOI: https://doi.org/10.33545/26164485.2021.v5.i2d.386

\begin{abstract}
Epilepsy is a chronic non-communicable neurologic condition that affects all ages of people by enduring predisposition to generate seizures, unprovoked by any immediate central nervous system insult and by neurobiologic, cognitive, psychological and social consequences of seizure recurrences. In most of the cases, the cause of epilepsy remains unknown and undetermined. However a few factors such as head injury, infections, tumor in brain, stroke, substance abuse and hereditary factors are the probable causes. Most of the people with epilepsy facing premature death is upto three times higher than for the general population. Three quarters of people with epilepsy living in low income countries don not get treatment they need. This article provides an overview of seizures focusing on epileptogenesis, mechanisms, clinical details, therapeutic approaches from different sources. Homoeopathy focuses on individualization of patient with holistic approach thus helps in managing epileptic seizures on dynamic plane.
\end{abstract}

Keywords: epilepsy, ILAE classification, homoeopathy

\section{Introduction}

Epilepsy being an emergency situation affects over 50 million people worldwide and over 10 million people in India ${ }^{[1]}$. Its prevalence is about $1 \%$ in Indian population, which is almost three times higher in rural population compared to the urban population ${ }^{[2]}$.

Epilepsy is a disease of the brain with either: (1) at least two unprovoked (or reflex) seizures occurring more than $24 \mathrm{~h}$ apart; (2) one unprovoked seizure or reflex seizure and a probability of further seizures similar to the general recurrence risk (at least $60 \%$ ) after two unprovoked seizures, occurring over the next 10 years; (3) diagnosis of an epilepsy syndrome ${ }^{[3]}$.

\section{Epidemiology}

The WHO estimates that there are 50 million people living with epilepsy (PWE) worldwide, making it a universal global neurological problem ${ }^{[19]}$. There are around 10-12 million people with epilepsy reside in India ${ }^{[19]}$. The incidence of status epilepticus has a bimodal age distribution with peaks during infancy and also in elderly age group. As per study in 2010 on epilepsy, from Kolkata, estimated an age-standardized incidence rate of 27.3/100,000 per year ${ }^{[20]}$. The incidence rate ranges approximately between 7 to 40 cases per 100,000 persons/year in recent times ${ }^{[18]}$. Around 30\% Women with Epilepsy report an increased propensity for seizures during menstruation, a phenomenon referred to as catamanial epilepsy. A study observed that the risk of seizure was increased during the ovulatory than the anovulatory phase ${ }^{[21]}$.

\section{Patho-physiology}

Status-epilepticus is a neurologic emergency condition with a time-dependent impact on both mortality and morbidity. Generalized convulsive status-epilepticus can be easily diagnosed as patients initially have generalized convulsions without return to neurologic baseline. However, as motor convulsions proceed, patients may cease to have overt motor features and status-epilepticus may only manifest as subtle jerks of the face, eyes, and extremities.

Non-convulsive status-epilepticus, which may have no clinical manifestations and is only detectable through video-EEG monitoring, is also increasingly being recognized. In all forms, prognosis is dependent on the underlying etiology and time period in statusepilepticus. Making the diagnosis is critical and not always straightforward, especially in the 
critical care setting, when seizures most often occur without apparent clinical signs. Preventing complications of statusepilepticus and its treatment and maintaining patients' overall health during and after the treatment of statusepilepticus contribute greatly to reducing patient morbidity and mortality, but often present multiple challenges that should not be overlooked.

Status epilepticus presents the persistence of abnormal excitation and the ineffective recruitment of inhibition. Excitation can come from many sources, such as an established epileptogenic circuit from preexisting epilepsy, excitation from the region surrounding a structural lesion, or diffuse excitation from a toxic/metabolic state.

These limbic and cortical inputs feed into the perforated pathway along the parahippocampal gyrus and to the neurons in the dentate gyrus. The dentate is the brake for excitatory activity frequently but when overwhelmed, excitatory activity feeds back to the hippocampus and then back to the parahippocampal gyrus, creating a selfamplifying reverberating circuit that perpetuates status epilepticus.

This patho-physiology can often be suspected when the brain MRI of a patient in status epilepticus shows a low level of restricted diffusion in the medial temporal structures on diffusion-weighted imaging. This MRI change may also present as "cortical ribboning" in focal status epilepticus. This restricted diffusion is reversible typically in 2 to 5 days after the seizure activity is terminated. Understanding the patho-physiology of status-epilepticus may lead to future treatment options. The first and very rapid changes in statusepilepticus involve protein phosphorylation, opening and closure of ion channels and release of neurotransmitters and modulators. Essentially all current antiepileptic drugs (AEDs) work at this level by either interacting with ion channels or possibly affecting the release of neurotransmitters in synaptic vesicles. Later changes in status epilepticus affect receptor trafficking with inhibitory receptors being removed from the synaptic cleft and excitatory neurotransmitters being reinforced.

Gamma aminobutyric acid (GABA) receptors are endocytosed into clathrin-coated pits and degraded in endosomes with progressive seizures. Glutamate receptors are recruited to the synaptic cleft and likely play a role in the mal-adaptive changes that perpetuate status-epilepticus. With increasing time spent in status epilepticus, additional changes involve neuro-peptide modulators. Poorly understood processes lead to continued seizures and eventually spontaneous epileptogenicity.

\section{Etiology}

Since $50 \%$ cases of status epilepticus involve an acute symptomatic etiology (e.g., hemorrhage, stroke, encephalitis) and others include exacerbations of chronic etiologies (e.g., tumor, remote stroke, traumatic brain injury), addressing the underlying etiology must occur concurrently with terminating the seizure activity. For example, a sub-dural hematoma that has caused cerebral edema and seizures may proceed to herniation without prompt intervention. Simply stopping the seizures in this case would not benefit the patient, and the seizures would likely stay refractory until the hematoma is evacuated and the cerebral edema and hydrocephalus controlled.

Patients with underlying metabolic encephalopathies are also at risk for status epilepticus, and identifying non- convulsive seizures on continuous EEG is the major diagnostic challenge. If the seizures are not recognized, the patient may have a worse cognitive outcome because of prolonged non-convulsive status epilepticus. Until the underlying cause of the metabolic disturbance (e.g., sepsis, liver failure) is corrected, the seizures are unlikely to respond to treatment. Patients with an autoimmune or paraneoplastic etiology may also continue to have refractory seizures until the underlying condition is addressed.

Meningitis, head injury or trauma, stroke, brain tumor, high fever (Febrile Seizure), and parasite infection Neurocysticercosis are the etiological factors. The main triggering factors are light, noise, sleep loss, alcohol intake, and cigarette smoking.

\section{Potential acute etiological factors ${ }^{[17]}$ Include}

- CNS (Central nervous system) infections (e.g., meningitis, encephalitis, and intracranial abscess)

- Metabolic abnormalities (hypoglycemia, hyponatremia, hypocalcemia, hepatic encephalopathy, and inborn errors of metabolism in children)

- Cerebro-vascular accidents

- Head trauma (with or without intracranial bleed)

- Drug toxicity

- Drug withdrawal syndromes (i.e., alcohol, benzodiazepines, and barbiturates)

- Hypoxia

- Hypertensive emergency

- Autoimmune disorders

\section{Classification}

The simplest way to classify status epilepticus is as convulsive or non-convulsive. However, the motor activity with seizures is a dynamic process and these two classes are not completely separate. Generalized convulsive status epilepticus has pronounced convulsive motor activity that typically involves tonic contractures followed by clonic jerking that are classically recognized as seizure activity.

These generalized convulsive activities are classified as generalized convulsive status epilepticus if they persist for more than 5 minutes. After a period of time spent in generalized convulsive status epilepticus, the body is no longer able to produce intense motor activity and instead generates more subtle jerks of the face, eyes, and extremities. With the less-intense motor activity, the seizures are typically called non-convulsive, although they are often identical to generalized convulsive status epilepticus on EEG recordings.

Classifying the mental status of a patient in status epilepticus is a similar challenge. If a conscious patient suddenly becomes unconscious with a seizure, the ictal phenomenon is easily recognized as a seizure with altered awareness. However, if a comatose patient develops the same electrographic seizure, it is often referred to as "seizures with no clinical signs" because the patient's preexisting poor mental status complicates the ascertainment of a seizure-specific alteration of consciousness.

Despite these challenges, the classification of convulsive and non-convulsive seizures is important. Convulsive status epilepticus appears to be more rapidly damaging to the patient and should therefore be aggressively treated. Being aware of the subtleties of non-convulsive status epilepticus provides a critical heightened awareness for this diagnostic 
possibility. Without such increased attention, nonconvulsive status epilepticus can be completely missed if not suspected and then looked for on continuous EEG monitoring.

\section{ILAE 2017 classification of seizure types expanded version ${ }^{[7]}$}

International League against Epilepsy (ILAE) 2017 classification has categorized seizures based on three key features: the location of seizure onset, level of awareness during a seizure, and other features of seizures ${ }^{[7]}$.

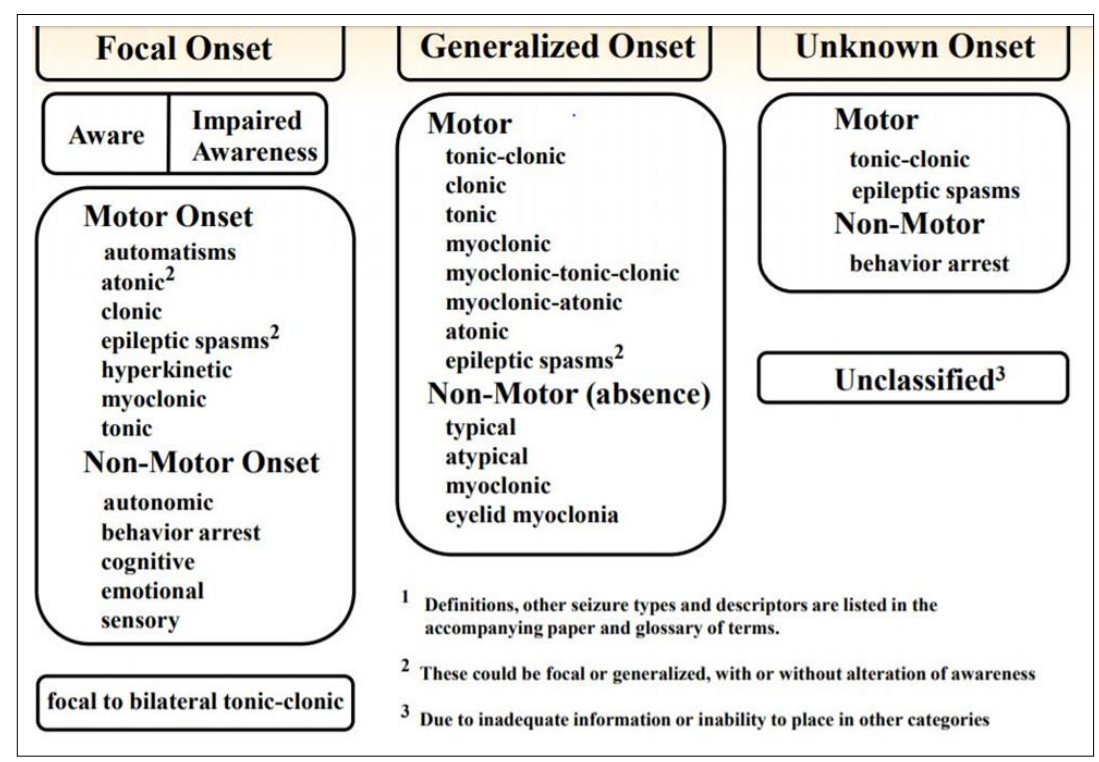

This new ILAE classification allows new types of focal seizures and a few new generalized seizures, and clarifies terms used to name seizures ${ }^{[8]}$.

Seizures of Temporal Lobe Origin are the most common type of focal impaired awareness (Complex partial) seizures. Frontal Lobe origin constitutes 30\%. Seizures arising from the insular region can mimic frontal, temporal, parietal lobe seizures ${ }^{[9]}$.

\section{International classification of seizures 1981}

Partial seizures (start in one place)

- Simple (no loss of consciousness of memory)

- Sensory Motor

- Sensory-Motor Psychic (abnormal thoughts or perceptions)
- Autonomic (heat, nausea, flushing, etc.)

- Complex (consciousness or memory impaired)

- With or without aura (warning)

- With or without automatisms

- Secondarily generalized

Generalized seizures (start over wide areas of brain)

- Absence (petit mal)

- Tonic-clonic (grand mal)

- Atonic (drop seizures)

- Myoclonic

\section{Other unclassifiable seizures}

Table 1: New terms introduced in the ILAE seizure classification, 2017

\begin{tabular}{|ll|}
\hline Old terms & New terms \\
\hline Partial & Focal \\
Simple partial & Focal aware \\
Complex partial & Focal impaired awareness \\
Psychic & Cognitive \\
$\begin{array}{l}\text { Secondary generalized } \\
\text { tonic- clonic }\end{array}$ & Focal to bilateral tonic-clonic \\
Arrest, Freeze, Pause, & Behavior arrest \\
Interruption & \\
Dyscognitive & Focal impaired awareness \\
Astatic & (Focal or generalized) atonic \\
Grand mal & Generalized tonic clonic, Focal to \\
& bilateral tonic clonic, Unknown \\
& onset tonic clonic \\
Infantile spasm & Epileptic spasm \\
Psychomotor & Focal impaired awareness \\
\hline
\end{tabular}


According to a study, the ILAE 2017 seizure classification demonstrated a steady transition from the 1981 International classification with acceptable consistency and improvements ${ }^{[10]}$.

\section{Diagnosis}

The diagnosis of generalized convulsive status epilepticus is fairly straight forward and based on clinical seizure activity. Generalized status epilepticus should be attended without any delay. Once some type of treatment has been initiated and the intense motor convulsions are controlled, the etiology of the status epilepticus should immediately be sought.

The most common etiologies include seizure breakthroughs in the context of a known chronic epilepsy, acute structural lesions, or acute metabolic derangements. Non-convulsive seizures present a much more difficult diagnostic challenge. Patients with altered mental status in the critical care setting have a risk for non-convulsive seizures that is proportional to the severity of the altered mental status. Patients in coma have the highest risk of non-convulsive status epilepticus at $30 \%$ to $40 \%$. Finding a patient with altered mental status greater than expected for his or her underlying condition should raise concern for non-convulsive status epilepticus. Patients with primary neurologic pathology (eg, hemorrhage, tumor, stroke, central nervous system, infection, and anoxia) have the highest risk of seizures.

The typical first step in diagnosis to evaluate high-risk primary neurologic pathology, CT scan of Head is needed. MRI Brain is required to evaluate whether the full range of structural pathology that could be the cause for seizures or status epilepticus, but given the length of the procedure, it may be deferred until the status epilepticus is adequately treated. If any concern of infection, possible meningitis, or encephalitis exists, a lumbar puncture should be performed, but it should also not delay initial treatment.

Patients at risk for status epilepticus based on clinical criteria (e.g., altered mental status, high-risk neurologic pathology, subtle clinical jerks) should be urgently recorded on continuous EEG monitoring; guidelines have been established for this monitoring. Specific tRNA fragments may constitute a novel class of epilepsy biomarker that could support the prediction of seizure risk in patients diagnosed with epilepsy ${ }^{[11]}$.

\section{Treatment and Management}

Epilepsy must be attended quickly and in an organized manner, with simultaneous assessment besides management of the airway, breathing, and circulation (ABC monitoring), while also administering antiepileptic drug (AED) treatment. The primary goal of management is to definitively terminate seizure activity as rapidly as possible while supporting the patient's cardiovascular and respiratory status. A significant portion of People with Epilepsy do not receive appropriate treatment, leading to a large treatment gap.

Lorazepam (Benzodiazepines) are the antiepileptic drug of choice for emergent control. It is preferred because of its rapid onset of action [21]. Intravenous (IV) infusion of midazolam, pentobarbital, thiopental, or propofol is helpful in Refractory Status Epilepticus; of that propofol must not be given to children. Choices include fosphenytoin, phenytoin, levetiracetam (40 to 60 and valproic acid can be concurrently used with benzodiazepines. In pregnant women with eclampsia, Magnesium sulphate will be helpful ${ }^{[21]}$.

For Post Stroke Seizure, prevention and prophylactic treatment, although some studies advocated possible shortterm prophylactic antiepileptic treatment for $\mathrm{ICH}$, the American Heart Association and the European Stroke Organization have stated that prophylactic administration of AEDs to prevent a seizure is not recommended for patients with stroke, including patients with $\mathrm{ICH}^{[12]}$.

\# Phytocannabinoids have demonstrated therapeutic efficacy across numerous neurological disorders ${ }^{[13]}$. As per a recent research study, Phytocannabinoids especially (CBD) Cannabidiol can be an approved therapy to treat epilepsy; except for seizure reduction, it has also been demonstrated that these phytocannabinoids affect neurogenerative and protective processes due to their inherent ability to modulate ECS signaling ${ }^{[14]}$.

- Cannbidiol CBD is a chemical component of the Cannabis sativa plant, more commonly known as marijuana. Homeopathically Cannabis indicus and $C$. sativus been already in usage from Hahnemannian era in potentised form for some cases of epilepsy with which we can reduce side effects and give individualized complete treatment based on symptomatology.

\section{Homoeopathic approach}

Homeopathic system is a holistic system of therapy but since its inception, it received sever criticism from scientific community due to non-measurable amount of drug substance in the homeopathic dose. Still homoeopathy has benefited globally, millions and billions of people, a fact that does not require any citation. In case of epileptic management, there are several homeopathic remedies reported in reported in scientific publications as well as homeopathic literature.

\section{Cicuta: Where there are distortions of body}

Cicuta is one of the best medicines for epilepsy where the attacks of convulsions are marked by violent, distorted body shape. The distortions are most frightful. There is present a marked opisthotonus which refers to the condition where the spine bends backward with the head and heels touching the ground. The person is totally unconscious. Cicuta is also best known for epilepsy following head injury. Cicuta can be used for convulsions occurring in children during dentition, in women during and after delivery and also for convulsions due to worms. Violent body distortions were seen. Touch and noise are the triggering factors for using Cicuta are. An auditory aura may show its presence.

\section{Artemisia vulgaris: For epilepsy with petit mal seizure}

Artemisia Vulgaris is the best natural medicine in case of Petit Mal Epilepsy especially in children. The main symptom is frequent brief episodes of seizures in a short time period. Ailments from strong emotions, including fear can cause convulsions. Absence of aura i.e., any subjective feeling predictive of the onset of a seizure prior to an attack is marked.

\section{Stramonium: For epileptic convulsions triggered by bright light}

Stramonium is the most suitable when the convulsion arises after exposure to bright light or shining objects. The consciousness is preserved and jerking of muscles of the 
upper body part is experienced by the patient.

Belladonna: Convulsions during teething, with fever; comes on suddenly, head hot, feet cold rush of blood to head and face

As per a previous study on dogs, considerable reduction was seen in seizure activity with belladonna, gradually there was disappearance of fits with continuous therapy ${ }^{[16]}$.

A wide range of fruitful benefits were being observed on Animals and pets from Hahnemannian era to till date ${ }^{[15,16]}$.

\section{Cuprum met: When aura for seizure attack is felt in knees}

Cuprum Met is the best suitable medicine when the seizure attack is preceded by an aura in the knee. The symptoms marked during the attack are clonic spasm that usually begins in finger or toes and soon covers the entire body. Jerking of muscles is also noticed. The Ailments from fright and anger can cause epilepsy. In females, Cuprum Met can be used in the treatment of convulsion during pregnancy, after delivery and around the menstrual period.

Staphysagria: Ailments from anger and humiliation, sexual passion, nervous excitability, sensitivity to insults. Ailments after indignation, grief, anger

Nervous affections with marked irritability; violent outbursts of passion, hypochondriacal, sad. Stitches flying to the ear on swallowing especially left.

\section{Bufo rana: For epileptic fit during sleep}

Bufo Rana is helpful in providing a cure for Epilepsy seizures where the attacks are present during sleep. The aura is felt in the genital area. It also works well for females who have attacks of seizures during menses (catamanial epilepsy).

\section{Hyoscyamus: For deep sleep following epileptic attack}

The best suitable when deep sleep follows an epileptic fit is Hyoscyamus. The other symptoms include picking at bedclothes and playing with hands and muscular twitching.

Homeopathic remedy nux vomica has also been reported to affect entire gray matter of the cerebrospinal nervous system (CNS), especially centering upon tubular gray matter of pons, medulla, and cervical portion of spinal cord

As per study conducted to investigate the antiepileptic effect of homeopathic remedy Nux vomica on mice and its comparison with standard therapeutic diazepam-clearly indicated the efficacy of Nux vomica on strychnine induced neurological disorder leading to death. It was found statistically significant in protecting the animals. This study made a pavement for further research on the mechanism of action of this medicine ${ }^{[5]}$

\section{Conclusion}

The prognosis and quality of life of a person with epilepsy varies considerably. It depends on the type and severity of the seizures, response to drug treatment, co-existing developmental and cognitive disorders, other comorbidities and the occurrence of episodes of status epilepticus. Though a large number of anti-epileptic drugs that supress or prevent the seizures cure is completely unavailable. We have no treatments that prevent the development of epilepsy or modify the detrimental course of the disorder. In contrast, treating epilepsy with homoeopathy will focus on clinical history of the individual and produce maximum therapeutic effect. Art of prescription in homoeopathy is done by considering triggers i.e., maintaining and exciting causes which helps to prevent recurrence of epilepsy. Most appropriate medicine is derived only after careful assessment of physical and psychological symptoms which is administered in highly diluted doses. Being holistic model of treatment, homoeopathy serves mankind without posing any risk of side effects and helps to reduce the dependence of conventional medicines.

\section{References}

1. Sridharan R, Murthy BN. Prevalence and pattern of epilepsy in India. Epilepsia 1999;40(5):631-6.

2. Leonardi M, Ustun TB. The global burden of epilepsy. Epilepsia 2002;43(6):21-5.

3. Curr opin Neurol. Department of Neurology and Neurological Sciences, Stanford University School of Medicine, Stanford, California, USA 2015;28(2):130-5. doi: 10.1097/WCO.0000000000000174.

4. Pharmacogn J. Antiepileptic Effect of Nux vomica, Homeopathic Remedy, Against Strychnine-Induced Seizers Anjana Goel*, Aditya Saxena, Ashok Kumar Bhatia 2018;10(2):245-248.

5. Goel A, Saxena A, Bhatia AK. Antiepileptic Effect of Nux Vomica, Homeopathic Remedy, Against Strychnine Induced Seizers. Pharmacog J 2018;10(2):245-8.

6. $\mathrm{Xu}$ MY. Poststroke seizure: optimizing its management. Stroke Vasc Neurol 2019;4(1):48-56.

7. Fisher RS, Cross JH, D'Souza C et al. Instruction manual for the ILAE 2017 operational classification of seizure types. Epilepsia 2017.

8. Robert Fisher S. The New classification of seizures by International League against Epilepsy 2017: Cur Neurol Neurosci. Rep 2017;17(6):48.

9. Anil Kumar, Sandeep Sharma. Complex Partial Seizure; N Treasure Island (FL): Statpearls Publishing 2021.

10. Hui Gao, Josemir Sander W, Yingfeng Xiao, Yingying Zhang, Dong Zhou. A comparison between the 1981 and 2017 International League Epilepsy classification of seizures types based on an outpatient setting; Epileptic Disord 2018;20(4):257-264.

11. Sengoku A. The contribution of JH Jackson to presentday epileptology. Epilepsia 2002;43(S9):6-8.

12. Michael Xu Y. Post stroke seizure: optimizing its management; Stroke Vasc Neurol 2019;4(1):48-56

13. Friedman D, French JA, Maccarrone M. Safety, efficacy, and mechanisms of action of cannabinoids in neurological disorders. Lancet Neurol 2019;18:504512.

14. Aaron Farrelly M, Styliani Vlachou. Konstantinos Grintzalis. Efficacy of Phytocannabinoids in Epilepsy treatment: Novel approaches and recent advances; Int. J Environ. Res. Public Health 2021;18(8):3993.

15. John Saxton. Homoeopathic treatment of Primary or Idiopathic seizures; Innovative veterinary Care 2017.

16. Varshney JP. Clinical management of idiopathic epilepsy in dogs with homoeopathic Belladonna 200C: a case series; Case Reports: Homoeopathy 2007;96(1):46-8. 
17. Langenbruch L, Krämer J, Güler S, Möddel G, Geßner $\mathrm{S}$, Melzer $\mathrm{N}$ et al. Seizures and epilepsy in multiple sclerosis: epidemiology and prognosis in a large tertiary referral center. J Neurol 2019.

18. Todd Wylie, Divya Jot S, Sandhu, Najib Murr. Status Epilepticus; Treasure Island (FL). Reddy SR. Effect of homoeopathic medicine Lycopodium clavatum in urinary calculi. International Journal of Applied Research 2017;3(1):790-1. Statpearls publishing 2021.

19. Garg D. Specific considerations for epilepsy in India. Curr Med Issues 2020;18:105-10.

20. Banerjee TK, Ray BK, Das SK, Hazra A, Ghosal MK, Chaudhuri A et al. A longitudinal study of epilepsy in Kolkata, India. Epilepsia 2010;51:2384-91.

21. Thomas SV, Sarma PS, Nirmala C, Mathai A, Thomas SE, Thomas AC. Women with epilepsy and infertility have different reproductive hormone profile than others. Ann Indian Acad Neurol 2013;16:544-8. 